\title{
Understanding missed opportunities for more timely diagnosis of cancer in symptomatic patients after presentation
}

\author{
G Lyratzopoulos ${ }^{\star 1,2}$, P Vedsted ${ }^{3}$ and H Singh ${ }^{4}$
}

${ }^{1}$ Health Behaviour Research Centre, Department of Epidemiology and Public Health, University College London, 1-19 Torrington Place, London WC1E 6BT, UK; ${ }^{2}$ Department of Public Health and Primary Care, Cambridge Centre for Health Services Research, University of Cambridge, Institute of Public Health, Forvie Site, Robinson Way, Cambridge CB2 0SR, UK; ${ }^{3}$ Department of Public Health, Research Unit for General Practice, Research Centre for Cancer Diagnosis in Primary Care (CaP), Aarhus University, DK-Bartholins Allé, 8000 Aarhus, Denmark and ${ }^{4}$ Houston Veterans Affairs Center for Innovations in Quality, Effectiveness and Safety, Michael E. DeBakey Veterans Affairs Medical Center and the Section of Health Services Research, Department of Medicine, Baylor College of Medicine, Houston TX 77030, US

\begin{abstract}
The diagnosis of cancer is a complex, multi-step process. In this paper, we highlight factors involved in missed opportunities to diagnose cancer more promptly in symptomatic patients and discuss responsible mechanisms and potential strategies to shorten intervals from presentation to diagnosis. Missed opportunities are instances in which post-hoc judgement indicates that alternative decisions or actions could have led to more timely diagnosis. They can occur in any of the three phases of the diagnostic process (initial diagnostic assessment; diagnostic test performance and interpretation; and diagnostic follow-up and coordination) and can involve patient, doctor/care team, and health-care system factors, often in combination. In this perspective article, we consider epidemiological 'signals' suggestive of missed opportunities and draw on evidence from retrospective case reviews of cancer patient cohorts to summarise factors that contribute to missed opportunities. Multi-disciplinary research targeting such factors is important to shorten diagnostic intervals post presentation. Insights from the fields of organisational and cognitive psychology, human factors science and informatics can be extremely valuable in this emerging research agenda. We provide a conceptual foundation for the development of future interventions to minimise the occurrence of missed opportunities in cancer diagnosis, enriching current approaches that chiefly focus on clinical decision support or on widening access to investigations.
\end{abstract}

The diagnosis of cancer in symptomatic patients requires a complex, multi-step process. Consequently, some patients experience prolonged intervals to diagnosis, which arise from various patient, doctor and health-care system-related factors involved in this process. Prompt diagnosis in symptomatic cancer patients represents a core deliverable of modern health-care systems, and the public (including patients, politicians and the media) considers it a serious priority. However, tensions between societal expectations for prompt cancer diagnosis in all patients and the challenges involved in achieving this aim are being increasingly recognised by patient groups, health-care professionals and policy makers. Research on strategies to minimise prolonged diagnostic intervals after presentation has become a priority. The objectives of this article are to highlight factors involved in missed opportunities for cancer diagnosis among symptomatic patients and discuss potential mechanisms and approaches to accelerating progress towards minimising diagnostic delays post presentation. We provide a conceptual foundation for developing multi-faceted strategies to achieve timely diagnosis in the greatest possible numbers of patients post presentation.

\section{RETROSPECTIVE CASE ANALYSES SUGGESTIVE OF MISSED DIAGNOSTIC OPPORTUNITIES \\ A large body of evidence based on analysis of the clinical details of cohorts of cancer patients suggests that 'missed opportunities'}

*Correspondence: Dr G Lyratzopoulos; E-mail: gl290@medschl.cam.ac.uk

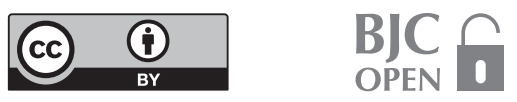




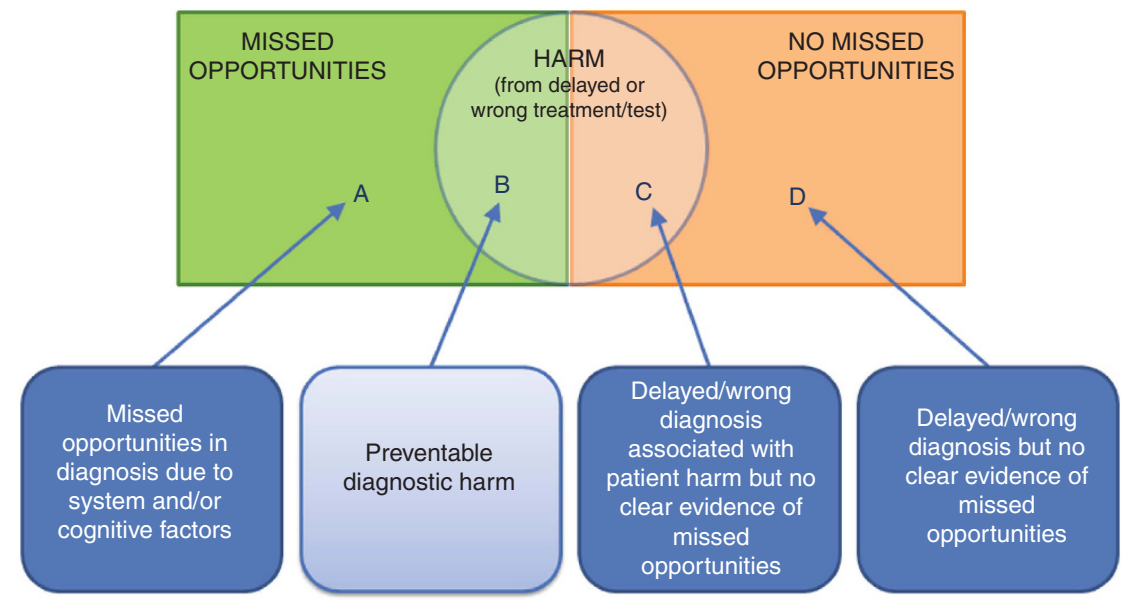

Figure 1. A model for defining missed diagnostic opportunities. Adopted from Singh, 2014.

occur in substantial proportions of patients (Singh et al, 2007, 2009a, 2010). A related concept is that of 'quality deviations' in the diagnosis of cancer (Jensen et al, 2014). A recent UK study also documents missed opportunities (Mitchell et al, 2013). Missed opportunities are instances in which post-hoc judgement indicates that alternative decisions or actions could have led to more timely diagnosis-i.e., something different could have been done or considered under the given circumstances to reach a more prompt diagnosis (Singh, 2014). For example, absence of evaluation for possible gastrointestinal bleeding in a 50-year-old man with new onset iron deficiency anaemia could represent a missed diagnostic opportunity for more timely diagnosis of gastrointestinal cancer. Determining the presence of missed opportunity involves a process of retrospective adjudication typically based on patient record audits, but it helps uncover critical areas for improvements in diagnostic quality.

It should be emphasised that not all missed opportunities or delays necessarily result in harm or poor patient outcomes and not all instances of delayed diagnosis are associated with missed opportunities (Figure 1; Singh, 2014). Missed opportunities may relate to any disease; for example, six out of seven patients

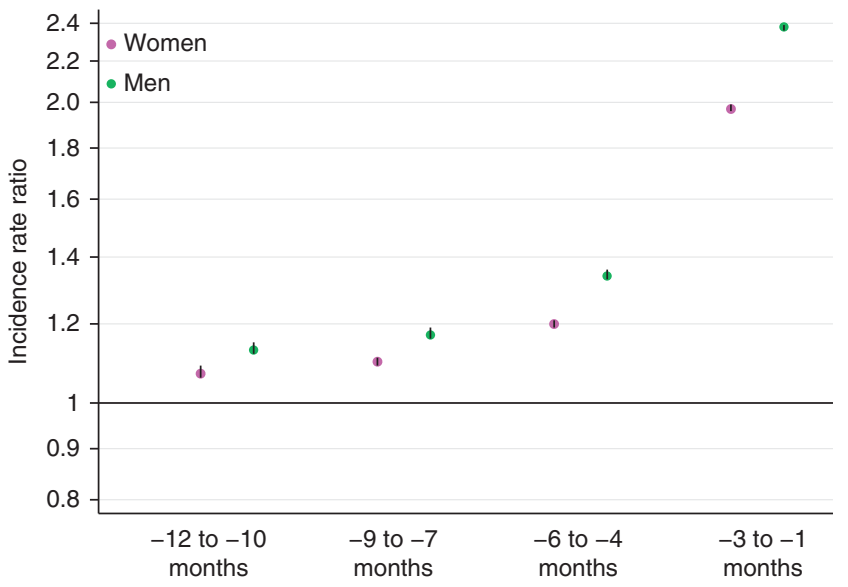

Figure 2. Epidemiological evidence suggestive of likely missed opportunities. Incidence rate ratios (IRR) for general practitioner consultations before the diagnosis of cancer compared with age- and sex-matched 'control' patients (without a diagnosis of cancer). Data from Christensen et al (2012); $n$ (women) = 63362 cancer patients and 633620 controls; $n$ (men) $=63848$ cancer patients and 638480 controls. Note very narrow $95 \%$ confidence intervals that exclude parity (i.e., 1.00); and excess risk spanning a 12-month period, including -6 to -4 months. subsequently diagnosed with chronic obstructive pulmonary disease are reported to have experienced missed opportunities, and very long diagnostic delays are reported among patients with ankylosing spondylitis (Hamilton et al, 2011; Jones et al, 2014). In this paper nonetheless we focus solely on patients with cancer who have sought medical help for their symptoms and exclude the consideration of potential opportunities for earlier presentation or participation in screening programmes.

\section{EPIDEMIOLOGICAL SIGNALS OF MISSED DIAGNOSTIC} OPPORTUNITIES

Retrospective evaluation of individual cases is not the only source of evidence on missed diagnostic opportunities. In England, work conducted by the (former) National Patient Safety Agency has highlighted factors implicated in diagnostic delays in cancer and recommended 'routine monitoring of delayed diagnosis' (NPSA, 2010). Although thus far no system that allows such routine monitoring exists, epidemiological evidence from England indicates that about one in five patients seen in general practice and subsequently diagnosed with cancer consults with their general practitioners three or more times for relevant symptoms before a specialist referral is made and that instances of multiple consultations are associated with prolonged primary care intervals (Lyratzopoulos et al, 2012, 2013a). Similarly, evidence from Denmark indicates that the rate of primary care consultations, diagnostic tests and hospital visits among patients subsequently diagnosed with cancer is substantially higher than that of 'control' patients (without cancer), over a period of several months before diagnosis (Figure 2) (Christensen et al, 2012; Ahrensberg et al, 2013; Hansen et al, 2015). However, epidemiological studies do not provide direct evidence or specific clinical information about the circumstances surrounding such events, and not all instances of these 'early'/multiple prediagnostic consultations would be associated with missed opportunities. For example, multiple consultations may be unavoidable in the presence of vague symptoms and/or when it is judged reasonable to investigate patients before referral (Lyratzopoulos et al 2014). Nonetheless, this type of evidence strongly indicates that missed opportunities may occur in at least some patients with cancer diagnoses and we need to understand more about their origins, both in primary and secondary care and throughout the diagnostic process. Further, epidemiological evidence can help identify cancer sites or socio-demographic characteristics of patients that confer a higher than average risk of delayed diagnosis (Lyratzopoulos et al 2012, 2013a), providing 
insights into potential responsible mechanisms and targets for further research and improvement initiatives.

TYPES AND ORIGINS OF MISSED OPPORTUNITIES IN CANCER DIAGNOSIS

Missed opportunities for diagnosing cancer sooner may occur anywhere in the diagnostic process. On the basis of evidence from retrospective case reviews of cohorts of cancer patients, missed opportunities typically occur in three main phases:

1. Initial diagnostic assessment (during the clinical encounter between a patient and a doctor, typically, but not exclusively, a generalist). This phase involves history taking, clinical examination and diagnostic reasoning, potentially also leading to specialist referral, test ordering or expectant ('safety netting'/ 'wait and see') management decisions, or their combination.

2. Diagnostic test performance and interpretation. This phase involves the process of performing appropriate diagnostic tests (e.g., blood tests, imaging or endoscopy, often at different times and locations) and their appropriate interpretation and associated actions.

3. Diagnostic follow-up and coordination. This phase includes many activities and tasks required to 'close the loop' on test results and referrals made on initial diagnostic assessment.

Patient, provider and system factors can all contribute to the generation of missed opportunities during one or more of the above phases, and missed opportunities in diagnosis often involve more than one contributory factor (Singh et al, 2013a). Complex interactions exist between these factors; for example, both patient and doctor factors could be influenced by system factors (Andersen et al, 2014). Understanding the complex interplay between these factors is important for reducing missed opportunities, thus underscoring the importance of using multi-disciplinary approaches in this area, including perspectives from psychology, human factors (the scientific field that focusses on how people interact with products, tools, procedures and processes) and informatics.

The concept of missed diagnostic opportunities builds on previous theoretical models from psychology. For example, the 'model of pathways to treatment' provides a holistic consideration of the journey from symptom onset to diagnosis and treatment initiation, encompassing four distinct intervals (symptom appraisal, helpseeking, diagnostic and pre-treatment intervals), with the diagnostic interval being of relevance to missed opportunities after presentation as considered in this paper. (Walter et al, 2012; Scott et al, 2013).

We use the high-level taxonomy of phases described above to illustrate different types of missed opportunities and related contributing factors to inform and motivate further policy initiatives and research.

\section{Missed opportunities and contributing factors during initial diagnostic assessment}

Rigid consultation norms. In some countries (including the UK and Denmark) medical consultation norms encourage patients to consult for 'one problem at a time' (which may even have to be declared in advance, before consultation), while the duration of primary care appointments is typically as short as $10 \mathrm{~min}$ (National Health Service Information Centre, 2007; Andersen et al, 2014; McCartney, 2014). Further, notable proportions of the public in countries with publicly funded health-care systems worry about consulting for symptoms that may 'waste the doctor's time' (Forbes et al, 2013). Beyond increasing the risk of delayed presentation and help-seeking, such attitudes might also decrease patient resolve to use up consultation time for communicating the full breadth and complexity of their symptoms, thereby increasing the risk of missed opportunities (Andersen et al, 2011).
Inadequate history taking and examination. For several reasons, the full spectrum, nature and duration of symptoms may not be elicited during a primary care encounter. Retrospective medical record reviews of patients diagnosed with cancer indicate that insufficient symptom elicitation or recording and ineffective doctor-patient communication may account for many instances of missed opportunities (Singh et al, 2009a; Jensen et al, 2014). Time pressures, either real or perceived, may impede doctors to obtain a thorough history or elicit clinical signs when present (Andersen et al, 2014; McCartney, 2014). Other factors described below might also contribute.

Language barriers. An increasing number of cancer patients in Europe and North America have limited proficiency in the first language of their resident country. In such circumstances, lack of interpretative support may impede effective patient-doctor communication, with some epidemiological evidence suggesting that suspecting the diagnosis of cancer is less prompt (i.e., requiring a greater number of pre-referral consultations) in older ethnic minority patients with symptoms (Lyratzopoulos et al, 2012).

Cognitive factors impeding optimal initial clinical assessment and reasoning. Firmly suspecting the diagnosis of cancer during a single clinical encounter is difficult, as symptoms and signs are rarely pathognomonic and may also be seen early in their development (Jones et al, 2007; Hamilton 2009; Jones et al 2009). Furthermore, as public awareness campaigns, by their very nature, encourage larger proportions of persons with symptoms to consult, the already low positive predictive value of symptomatic presentations in primary care for cancer may decrease further. A range of factors may, however, make diagnostic reasoning even more challenging. These include the following:

- Cognitive biases: several types of such biases exist, including anchoring bias (focusing exclusively on a single item of information), availability bias (over-reliance on already known or easily available information) and 'commitment to a steer' (i.e., initial diagnostic impressions), which can impede diagnostic reasoning (Kostopoulou et al, 2012; Croskerry 2013). In a study of diagnostic errors in UK primary care, biases at the initial framing of the problem were related to errors at the end of the diagnostic process (Balla et al, 2012). In addition to operating during the clinical encounter, these biases could also lead to the misinterpretation of diagnostic test results (Singh et al, 2012a).

- Co-morbidity: consideration of a cancer diagnosis is particularly challenging in the presence of other known non-cancer comorbid conditions. Many older patients (the age group at higher risk for cancer) are multi-morbid (Barnett et al, 2012). In these patients, symptoms compatible with the known cause of chronic morbidity could be easily thought to reflect the pre-existing disease rather than a new problem (Mitchell et al, 2013).

- Unfamiliarity with cancer presentations: patients with a new diagnosis of cancer are infrequent in general practice. For example, in the UK a full-time general practitioner on average might encounter only between 5 and 10 new cases in a year, amid thousands of patients with other conditions. Beyond unfamiliarity, 'epidemiological optimism' bias can make prompt suspicion of the diagnosis of cancer even harder in low-risk patient groups even when they complain of symptoms that may be due to cancer. Such groups include young persons and certain socio-demographic groups within specific cancers (e.g., women who present with visible haematuria; Lyratzopoulos et al, 2012, 2013b; Nicholson et al, 2014).

Access and system capacity constraints. These are often expressed as long waiting times. An indirect consequence of prolonged waiting times is that they de facto increase the disease severity threshold for referral decisions-i.e., capacity constraints influence doctor-decision making. This may be a particular challenge for publicly funded systems where demand management functions are implicitly delegated 
to primary care services (Vedsted and Olesen 2011; Brown et al, 2014). Geographical barriers, such as distance to diagnostic centres, may also be relevant, although evidence on such associations is needed.

Referral norms. The positive predictive value of signs and symptoms for cancer is low; only a few have values $>5 \%$ in patients presenting in general practice (Shapley et al, 2010). Consequently, most patients investigated for suspected cancer will not have the disease; for example, in the UK among patients who are referred to specialists for suspected cancer, about $90 \%$ will be found not to have cancer (Meechan et al, 2012). Likely peer pressure by specialists or hospital managers may increase reluctance by primary care doctors to refer patients if there is intolerance of low 'diagnostic hit rates', increasing the risk of missed opportunities, although evidence to further establish the role of such dynamics would be desirable.

\section{Missed opportunities and contributing factors during diagnostic test performance and interpretation}

Patient non-adherence with recommended tests and lack of system resilience towards such ('no show') events. Patients may not adhere to prescribed investigation plans, either by not attending recommended investigations or by not preparing for them. For example, patient non-adherence with suggested colonoscopy investigation can lead to post-referral diagnostic delays in colorectal cancer (Singh et al, 2012b). In Denmark, among all missed opportunities, $16 \%$ were attributable to patients not showing up (Jensen et al, 2014). Different factors may be implicated in 'no show' events. Evidence from patients not adhering to screening colonoscopy appointments implicates emotional barriers (such as fear of an adverse diagnosis, or fear of procedure-related pain or complications) and logistical or communication barriers (Denberg et al, 2005), and similar factors may be applicable to diagnostic colonoscopies. When 'no show' events do occur, communication with the patient and a review of the patient's management should be automatically triggered to reschedule investigations or initiate alternative management.

Diagnostic testing process complexity. The diagnosis of cancer typically requires a sequence ('chain') of tests and procedures (e.g., blood tests, imaging, endoscopy leading to tissue sampling and pathology reporting). These tests are by necessity often carried out at different locations and times, whereas generalist and specialist doctors often work in different settings, adding levels of complexity to the diagnostic process. This high degree of complexity multiplies the risk of delays and/or erroneous decision making at different steps in the process by a factor proportionate to the number of distinct tests required. Further, the distribution of the diagnostic process (in space and time, and between primary care and specialist care, often including different departments and locations) increases both time lags between different steps in the process and risks of miscommunication (Jensen et al, 2014), untimely communication or lack of follow-up of important test results. This has, for example, in Denmark, led to the formation of diagnostic units that enable the conduct of multiple required tests and specialist assessments 'within 1 day/under one roof, and similar services are currently being developed in the UK. Such strategies will need to be evaluated for their effectiveness. Earlier work by the National Patient Safety Agency has recommended that the health service needs to identify, review and disseminate current good practice in the process of ordering, managing and tracking tests and test results' (NPSA, 2010).

Inadequacies in the investigation strategy (initially negative tests in the presence of ongoing symptoms or diagnostic suspicion). This may occur when the suspicion of cancer is correctly raised but decisions on planned investigations are sub-optimal or inadequate (Jensen et al, 2014). This scenario may be more likely for cancers sharing many common symptoms (e.g., cancers of pelvic/ abdominal organs). For example, a patient with abdominal symptoms is investigated with a colonoscopy that is negative, and this finding is initially interpreted as bringing 'diagnostic closure', but the patient has persistent symptoms and is subsequently found to have cancer of another abdominal organ (pancreas, liver or ovary). Another example can be provided by false-negative chest X-ray findings in patients with suspected lung cancer (Bjerager et al, 2006; Stapley et al, 2006). Such circumstances can clearly prolong diagnostic intervals by providing (temporary) false reassurance (Singh et al, 2012a). A much more challenging situation occurs when correct tests have been carried out but the results are falsely interpreted as negative, without adequate fail-safe or back-up re-assessment mechanisms being present (Singh et al, 2012a; Middleton et al, 2014).

\section{Missed opportunities and contributing factors during follow-up and coordination}

Patient factors. If appropriately empowered, patients' active role in the diagnostic process can minimise the risks of missed opportunities. How this potential can be harnessed should be a priority for future research (McDonald et al, 2013). The National Patient Safety Agency report on diagnostic delays in cancer recommended that the health service 'develop methods for empowering patients on a cancer diagnostic pathway’ (NPSA, 2010). However, currently:

- Many patients do not feel empowered to seek out the results of tests performed on them, or do not know how to do so. Patients may also be 'reassured' by lack of follow-up by doctors/the health-care system, interpreting lack of communication to mean that 'all is normal' in instances when this is not the case. This emphasises the importance of passive ('open door') or active (fixed interval, e.g., 3-week clinical review) follow-up as part of safety-netting strategies (Almond et al, 2009).

- Patients also might not be willing to re-consult or seek a second medical opinion despite doubting the certainty of their diagnosis and persistent or worsening symptoms (Birt et al, 2014; McDonald et al, 2013).

- Another common occurrence is patients not returning for 'failsafe/safety-netting' visits planned as part of expectant management strategies, and/or when they experience persistent, worsening or new symptoms (Singh et al, 2012a; Mitchell et al, 2013). Factors similar to those involved in non-adherence with diagnostic investigations may be implicated (see 'Missed opportunities and contributing factors during diagnostic test performance and interpretation', above). Robust mechanisms for identification of such occurrences and contact with patients are required.

Over-reliance on patients to 'call back'. Doctors often believe that patients will call if they do not feel better or new symptoms develop, and often assume that the diagnosis they had recently given had been correct if they do not hear otherwise (Singh and Sittig, 2014). Ensuring timely patient follow-up could also help prevent missed opportunities that relate to coordination failures between different clinics, hospital departments and general practices (Mitchell et al, 2013). Proactive follow-up systems and protocols that leverage information technology might be needed to minimise the risk of such missed opportunities 'at the last hurdle' (Murphy et al, 2014).

Lack of appreciation or follow-up of abnormal test results. Increasingly recognised in 'electronic health record-enabled' health-care systems are instances of failure to recognise and act on abnormal tests related to cancer (Murphy et al, 2014). Many reasons could contribute to such occurrences-for example, physician 'alert fatigue' or ambiguities about the health-care professional who is 'in charge' of the patient and responsible for follow-up (Singh et al, 2009b). Informational continuity and clarity of accountability for the patient as they progress through the diagnostic pathway poses remarkable challenges (Press, 2014). Because modern health care is delivered by teams rather than by 
individuals, factors relating to team dynamics and 'distributed cognition' could also be relevant; these include low staff morale, poor communication between team members and limited 'situational awareness' of diagnostic safety (Singh et al, 2012a).

\section{MEASUREMENT, LEARNING AND IMPROVEMENT}

As is the case for safety interventions in general, identification of instances where missed opportunities have occurred is a pre-requisite for motivating and guiding organisational learning and improvement efforts and service redesign (Donaldson et al, 2014). Although we acknowledge that not all missed opportunities or delays would directly result in poorer patient outcomes (see also Figure 1), we believe that all preventable diagnostic delays should be avoided. Additional reasons to do so include strong patient (and carer) preferences for prompt diagnosis of cancer and substantial burden of patient complaints and medico-legal claims associated with diagnostic delays/missed diagnostic opportunities (Gandhi et al, 2006; Schiff et al, 2013).

Measurement of missed opportunities relies on operational definitions that may vary between settings and systems. Ideally, measures with optimal construct validity should be developed (Singh and Sittig, 2015). However, this is difficult to achieve given the current state of the evidence and scientific knowledge related to diagnostic safety. We therefore suggest that resources should be prioritised for developing and using 'surrogate markers' of missed opportunities, which could 'trigger' clinical audit activity and case reviews to verify the presence and nature of missed opportunities and likely contributory factors. Different possible surrogate markers (or triggers) have been proposed in the literature, including unusual patterns of multiple consultations ('return visits'), emergency presentations (Elliss-Brookes et al, 2012; Lyratzopoulos et al, 2012; Mitchell et al, 2013; Singh et al, 2013a; Jensen et al, 2014) or symptoms or abnormal test findings suggesting the need for diagnostic evaluation for cancer (Murphy et al, 2014). Another approach is doctor-led retrospective reviews of all or randomly selected cases of cancer diagnosed in a practice or health centre during defined periods (Baughan et al, 2009; Rubin et al, 2011; Mitchell et al, 2013). Patient record audits offer several advantages, including detailed information and longitudinal data about symptoms, investigations and diagnosis evolution, but their limitations include the potential for hindsight bias, missing documentation and review time (Zwaan et al, 2010). Although no criterion or method to define missed opportunities is $100 \%$ sensitive and specific, the use of markers/triggers can motivate and support quality improvement activities and professional and organisational learning.

We summarise common factors involved in missed opportunities and list a range of possible actions in Table 1, acknowledging that evidence on the effectiveness of cognitive and system interventions is still emerging and there is a need for further research and evaluation in relevant areas (Graber et al, 2012; Singh et al, 2012c).

IMPLICATIONS FOR RESEARCH AND POLICY. ENRICHING THE SPECTRUM OF POST-PRESENTATION INTERVENTIONS TO PROMOTE EARLY DIAGNOSIS

Recently, an Institute of Medicine (IOM) committee was tasked with evaluating diagnostic error as a patient safety issue (IOM, 2014). As this new report will be a continuation of the IOM's Health Care Quality Initiative report 'To Err is Human: Building a Safer Health System and Crossing the Quality Chasm: A New Health System for the 21st Century', the importance of early diagnosis and of improving diagnostic safety for both researchers and policymakers is likely to increase substantially in the coming years.

Several policies aimed at expediting the diagnosis of cancer after presentation to general practitioners chiefly emphasise either 'knowledge mobilisation' interventions (e.g., reminding primary care physicians of the possibility of cancer diagnosis during consultations), or enabling of greater access to endoscopy or imaging investigations (Department of Health, 2012; Hamilton et al, 2013). Although critically important, such interventions address only some of the multitude of factors along all phases of the diagnostic process that can contribute to missed opportunities in cancer diagnosis. In addition, they tend to focus on primary care rather than keeping a system-wide focus and addressing factors during the medical encounter as well as the performance of tests and follow-up and coordination beyond the initial clinical encounter. Further, in both primary and secondary care we are yet to understand why physicians (both generalists and specialists) at times do not recognise or act upon obvious alarm symptoms or signs of cancer. Factors such as doctors' tiredness or stress, information overload ('alert fatigue'), poor communication between primary and secondary care, and team dynamics are likely to be involved in such events (Singh et al, 2012a, 2013b). In contemporary complex and information technology-enabled health-care environments, acquiring such an understanding requires disciplinary inputs from the fields of psychology and behavioural/social sciences, human factors, systems engineering and clinical informatics. A systems approach to the problem of diagnostic safety is needed, as it is currently applied to surgical safety (Vincent et al, 2004). Interventions focussing only one factor-for example, solely aiming to optimise clinical reasoning during primary care encounters-might not be comprehensive enough, given that most instances of missed diagnostic opportunities typically involve several contributing factors.

We thus recommend that multi-disciplinary and multi-faceted approaches be developed to target the various phases in the diagnostic process where missed opportunities occur. These interventions should address the full range of contributing factors beyond mere individual cognitive or system capacity constraints. Because cancer diagnosis is distributed across time and place and involves interactions among multiple human and system components, these approaches should aim to strengthen both human and system performance and account for concepts such as shared mental models, distributed cognition and optimal technology use (Karsh et al, 2006; Henriksen and Brady, 2013; Smith et al, 2014). Newer forms of diagnostic and information technologies are being suggested to improve the diagnosis of cancer (e.g., electronic health records to enable informational continuity between different providers) requiring input from the field of clinical informatics (El-Kareh et al, 2013). However, although technological innovation can be helpful, many factors unrelated to technology can contribute to missed opportunities across all phases of the diagnostic process and must also be addressed. This can be achieved by a deeper understanding of 'socio-technical' factors implicated in missed opportunities (workflow and organisational factors among others; Sittig and Singh, 2010). Multi-faceted approaches will enrich the spectrum of intervention targets, beyond facilitation of symptom recognition or access to specialist diagnostic assessment.

In conclusion, we call for more multi-disciplinary research that targets factors contributing to missed opportunities in all phases of the diagnostic process. The fields of organisational and cognitive psychology, human factors science and clinical informatics can all provide valuable insights into this emerging research agenda. Building on current approaches, we have provided a theoretical basis for the development of future interventions to shorten diagnostic intervals post presentation. The conceptual foundation 


\section{Table 1. Future initiatives and research agenda to address missed opportunities}

Possible practical steps/policies

Future research agenda

Potential fields involved ${ }^{a}$

Re-engineering of medical consultation norms and improving the quality of history taking and examination

Allocation of additional consultation time, particularly for

infrequent attenders or multi-morbid patients (Andersen et al,

2014; McCartney, 2014)

Further quantification and qualification of

mechanisms leading to ineffective symptom

communication or elicitation during the

patient encounter, and its implications

Social science

Patient engagement initiatives bolstering confidence in communicating symptoms and in not feeling that they 'are wasting the doctors' time' (Andersen et al, 2011; Forbes et al, 2013)

Ensuring formal or informal (e.g., family/relative) translation services for patients who are not competent in routine consultation language (e.g., ethnic minority patients)

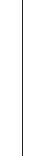

Behavioural science
Medical anthropology
Policy
Primary care

Health services research

Reducing cognitive or system barriers to optimal initial clinical assessment and reasoning

Effective decision support tools/diagnostic checklists to help minimise the risk of not thinking about a diagnosis of cancer in patients with common presentations and those with lower risk/ atypical symptoms (Ely et al, 2011; Hamilton et al, 2013)

Optimising decision making about investigation plans by increasing capacity/enabling prompt primary care access to specialist investigations and their prompt reporting

Further evaluation of the use and impact of clinical decision support tools and diagnostic checklists in randomised controlled trials

Evaluate the impact of additional consultation time, diagnostic services reorganisation, and

increasing capacity on measures of diagnostic Policy quality and safety
Clinical informatics

Cognitive psychology

Human factors

Primary care

Health services research

Cultural shift towards accepting 'low diagnostic hit rates'. Remodelling of diagnostic quality norms from kudos given to doctors for 'being right' to kudos given to doctors for 'being safe'

Improving diagnostic test performance, interpretation and follow-up

Development of resilient/fail-safe systems for following up and/ or rebooking patient 'no show' instances (for test performance or 'expectant management' follow-up), or patients with negative test results but persistent/evolving symptoms

Reorganisation of diagnostic pathways to allow for multiprocessing of tests in 'one-stop' clinics (all tests 'under one roof, during one day') and wider access to specialist investigations

Empowering of patients regarding outcomes of diagnostic investigations. Solutions may include sharing of patient records and enabling active chasing of test results by patients (Feeley and Shine, 2011)

Effective electronic health record-enabled systems to ensure that diagnostic tests with abnormal results are acted upon by the relevant clinician

Use of 'navigators' (volunteers or professional staff) for complex cases (e.g., multiple tests, high/worsening symptom burden, patients with poor social support and/or other communication or transport difficulties; (Press, 2014)

Robustly evaluate the effectiveness of new models of diagnostic care (Guldbrandt et al, 2014)

Explore optimal back-up tracking support systems for optimising diagnostic safety in electronic health record-enabled systems

Organisational
psychology
Human factors
Clinical informatics
Systems engineering
Behavioural science
Social science
Policy
Health services research
Nursing

Organisational

a This is an indicative list of relevant disciplines that should not be considered as 'future proof': input from scientific fields beyond those mentioned may also be needed, as knowledge in this area is evolving.

we provide could motivate multi-disciplinary and multi-faceted strategies aimed at minimising the occurrence of missed diagnostic opportunities in cancer, enriching current approaches that principally focus on clinical decision support or on widening access to investigations.

\section{ACKNOWLEDGEMENTS}

We acknowledge the helpful and incisive comments by Dr Rikke Sand Andersen (Aarhus University, Denmark) in conceptualising this piece and in drafts of the manuscript. The work is independent research supported by different funding schemes. GL was supported by a Post-Doctoral Fellowship by the National Institute for Health Research (PDF-2011-04-047) until the end of 2014 and by a Cancer Research UK Clinician Scientist Fellowship award (A18180) from 2015. HS is supported by the VA Health Services Research and Development Service (CRE 12-033; Presidential Early Career Award for Scientists and Engineers USA 14-274), the VA National Center for Patient Safety, the Agency for Health Care Research and Quality (R01HS022087) and in part by the Houston VA HSR\&D Center for Innovations in Quality, Effectiveness and Safety (CIN 13-413). PV was 
supported by $\mathrm{CaP}$, funded by The Danish Cancer Society and the Novo Nordisk Foundation.

\section{CONFLICT OF INTEREST}

The authors declare no conflict of interest.

\section{DISCLAIMER}

The views expressed in this publication are those of the authors and not necessarily those of the NHS, the National Institute for Health Research or the Department of Health, Cancer Researh UK, or any other funding organisation.

\section{REFERENCES}

Ahrensberg JM, Fenger-Grøn M, Vedsted P (2013) Use of primary care during the year before childhood cancer diagnosis: a nationwide population-based matched comparative study. PLoS One 8: e59098.

Almond S, Mant D, Thompson M (2009) Diagnostic safety-netting. Br J Gen Pract 59: 872-874.

Andersen RS, Tørring ML, Vedsted P (2014) Global healthcare seeking discourses facing local clinical realities: exploring the case of cancer. Med Anthropol Q. doi:10.1111/maq.12148.

Andersen RS, Vedsted P, Olesen F, Bro F, Søndergaard J (2011) Does the organizational structure of health care systems influence care-seeking decisions? A qualitative analysis of Danish cancer patients' reflections on care-seeking. Scand J Prim Health Care 29: 144-149.

Balla J, Heneghan C, Goyder C, Thompson M (2012) Identifying early warning signs for diagnostic errors in primary care: a qualitative study BMJ Open 2: e001539.

Barnett K, Mercer SW, Norbury M, Watt G, Wyke S, Guthrie B (2012) Epidemiology of multimorbidity and implications for health care, research, and medical education: a cross-sectional study. Lancet 380: 37-43.

Baughan P, O'Neill B, Fletcher E (2009) Auditing the diagnosis of cancer in primary care: the experience in Scotland. Br J Cancer 101(Suppl 2): S87-S91.

Birt L, Hall N, Emery J, Banks J, Mills K, Johnson M, Hamilton W, Walter FM (2014) Responding to symptoms suggestive of lung cancer: a qualitative interview study. BMJ Open Respir Res 1(1): e000067.

Bjerager M, Palshof T, Dahl R, Vedsted P, Olesen F (2006) Delay in diagnosis of lung cancer in general practice. Br J Gen Pract 56: 863-868.

Brown S, Castelli M, Hunter DJ, Erskine J, Vedsted P, Foot C, Rubin G (2014) How might healthcare systems influence speed of cancer diagnosis: A narrative review. Soc Sci Med 116C: 56-63.

Christensen KG, Fenger-Grøn M, Flarup KR, Vedsted P (2012) Use of general practice, diagnostic investigations and hospital services before and after cancer diagnosis-a population-based nationwide registry study of 127,000 incident adult cancer patients. BMC Health Serv Res 12: 224.

Croskerry P (2013) From mindless to mindful practice-cognitive bias and clinical decision making. N Engl J Med 368: 2445-2448.

Denberg TD, Melhado TV, Coombes JM, Beaty BL, Berman K, Byers TE, Marcus AC, Steiner JF, Ahnen DJ (2005) Predictors of nonadherence to screening colonoscopy. J Gen Intern Med 20: 989-995.

Department of Health (2012) Direct Access to Diagnostic Tests for Cancer Best Practice Referral Pathways for General Practitioners. https://www.gov.uk/government/uploads/system/uploads/attachment_ data/file/216503/dh_133511.pdf.

Donaldson LJ, Panesar SS, Darzi A (2014) Patient-safety-related hospital deaths in England: thematic analysis of incidents reported to a national database, 2010-2012. PLoS Med 11: e1001667.

Elliss-Brookes L, McPhail S, Ives A, Greenslade M, Shelton J, Hiom S, Richards M (2012) Routes to diagnosis for cancer-determining the patient journey using multiple routine data sets. Br J Cancer 107: 1220-1226.

El-Kareh R, Hasan O, Schiff GD (2013) Use of health information technology to reduce diagnostic errors. BMJ Qual Saf 22: ii40-ii51.
Ely JW, Graber ML, Croskerry P (2011) Checklists to reduce diagnostic errors. Acad Med 86: 307-313.

Feeley TW, Shine KI (2011) Access to the medical record for patients and involved providers: transparency through electronic tools. Ann Intern Med 155: 853-854.

Forbes LJ, Simon AE, Warburton F, Boniface D, Brain KE, Dessaix A, Donnelly C, Haynes K, Hvidberg L, Lagerlund M, Lockwood G, Tishelman C, Vedsted P, Vigmostad MN, Ramirez AJ, Wardle J. International Cancer Benchmarking Partnership Module 2 Working Group (2013) Differences in cancer awareness and beliefs between Australia, Canada, Denmark, Norway, Sweden and the UK (the International Cancer Benchmarking Partnership): do they contribute to differences in cancer survival? Br J Cancer 108: 292-300.

Gandhi TK, Kachalia A, Thomas EJ, Puopolo AL, Yoon C, Brennan TA, Studdert DM (2006) Missed and delayed diagnoses in the ambulatory setting: a study of closed malpractice claims. Ann Intern Med 145: 488-496.

Graber ML, Kissam S, Payne VL, Meyer AN, Sorensen A, Lenfestey N, Tant E, Henriksen K, Labresh K, Singh H (2012) Cognitive interventions to reduce diagnostic error: a narrative review. BMJ Qual Saf 21: 535-557.

Guldbrandt LM, Rasmussen TR, Rasmussen F, Vedsted P (2014) Implementing direct access to low-dose computed tomography in general practice-method, adaption and outcome. PLoS One 9: e112162.

Jensen H, Nissen A, Vedsted P (2014) Quality deviations in cancer diagnosis: prevalence and time to diagnosis in general practice. Br J Gen Pract 64: e92-e98.

Jones RC, Price D, Ryan D, Sims EJ, von Ziegenweidt J, Mascarenhas L, Burden A, Halpin DM, Winter R, Hill S, Kearney M, Holton K, Moger A, Freeman D, Chisholm A, Bateman ED. Respiratory Effectiveness Group (2014) Opportunities to diagnose chronic obstructive pulmonary disease in routine care in the UK: a retrospective study of a clinical cohort. Lancet Respir Med 2: 267-276.

Jones R, Charlton J, Latinovic R, Gulliford MC (2009) Alarm symptoms and identification of non-cancer diagnoses in primary care: cohort study. BMJ 339: b3094.

Jones R, Latinovic R, Charlton J, Gulliford MC (2007) Alarm symptoms in early diagnosis of cancer in primary care: cohort study using General Practice Research Database. BMJ 334: 1040.

Hansen PL, Hjertholm P, Vedsted P (2015) Increased diagnostic activity in general practice during the year preceding colorectal cancer diagnosis. Int J Cancer 5 January 2015 doi:10.1002/ijc.29418. [Epub ahead of print].

Hamilton L, Gilbert A, Skerrett J, Dickinson S, Gaffney K (2011) Services for people with ankylosing spondylitis in the UK-a survey of rheumatologists and patients. Rheumatology (Oxford) 50: 1991-1998.

Hamilton W (2009) The CAPER studies: five case-control studies aimed at identifying and quantifying the risk of cancer in symptomatic primary care patients. Br J Cancer 101(Suppl 2): S80-S86.

Hamilton W, Green T, Martins T, Elliott K, Rubin G, Macleod U (2013) Evaluation of risk assessment tools for suspected cancer in general practice: a cohort study. Br J Gen Pract 63: e30-e36.

Henriksen K, Brady J (2013) The pursuit of better diagnostic performance: a human factors perspective. BMJ Qual Saf 22: ii1-ii5.

IOM (Institute of Medicine) of the National Academies (2014) Diagnostic Error in Health Care. http://www.iom.edu/Activities/Quality/Diagnostic ErrorHealthCare.aspx.

Karsh BT, Holden RJ, Alper SJ, Or CK (2006) A human factors engineering paradigm for patient safety: designing to support the performance of the healthcare professional. Qual Saf Health Care 15(Suppl 1): i59-i65.

Kostopoulou O, Russo JE, Keenan G, Delaney BC, Douiri A (2012) Information distortion in physicians' diagnostic judgments. Med Decis Making 32: 831-839.

Lyratzopoulos G, Abel GA, McPhail S, Neal RD, Rubin GP (2013a) Measures of promptness of cancer diagnosis in primary care: secondary analysis of national audit data on patients with 18 common and rarer cancers. Br J Cancer 108: 686-690.

Lyratzopoulos G, Abel GA, McPhail S, Neal RD, Rubin GP (2013b) Gender inequalities in the promptness of diagnosis of bladder and renal cancer after symptomatic presentation: evidence from secondary analysis of an English primary care audit survey. BMJ Open 3: e002861.

Lyratzopoulos G, Neal RD, Barbiere JM, Rubin GP, Abel GA (2012) Variation in number of general practitioner consultations before hospital referral for cancer: findings from the 2010 National Cancer Patient Experience Survey in England. Lancet Oncol 13: 353-365. 
Lyratzopoulos G, Wardle J, Rubin G (2014) Rethinking diagnostic delay in cancer: how difficult is the diagnosis? BMJ 349: g7400.

McCartney M (2014) One problem. BMJ 348: g3584.

McDonald KM, Bryce CL, Graber ML (2013) The patient is in: patient involvement strategies for diagnostic error mitigation. BMJ Qual Saf 22(Suppl 2): ii33-ii39.

Meechan D, Gildea C, Hollingworth L, Richards MA, Riley D, Rubin G (2012) Variation in use of the 2-week referral pathway for suspected cancer: a cross-sectional analysis. Br J Gen Pract 62: e590-e597.

Middleton LP, Feeley TW, Albright HW, Walters R, Hamilton SH (2014) Second-opinion pathologic review is a patient safety mechanism that helps reduce error and decrease waste. J Oncol Pract 10(4): 275-280.

Mitchell ED, Rubin G, Macleod U (2013) Understanding diagnosis of lung cancer in primary care: qualitative synthesis of significant event audit reports. Br J Gen Pract 63: e37-e46.

Murphy DR, Laxmisan A, Reis BA, Thomas EJ, Esquivel A, Forjuoh SN, Parikh R, Khan MM, Singh H (2014) Electronic health record-based triggers to detect potential delays in cancer diagnosis. BMJ Qual Saf 23: $8-16$.

National Health Service Information Centre (2007) GP Workload Survey Results. www.hscic.gov.uk/pubs/gpworkload.

National Reporting and Learning Service, NPSA (National Patient Safety Agency) (2010) Delayed Diagnosis of Cancer: thematic Review. http:// www.nrls.npsa.nhs.uk/EasySiteWeb/getresource.axd?AssetID=69895\&.

Nicholson BD, McGrath JS, Hamilton W (2014) Bladder cancer in women. BMJ 348: g2171.

Press MJ (2014) Instant replay-a quarterback's view of care coordination. $N$ Engl J Med 371: 489-491.

Rubin GP, Elliott K, McPhail S (2011) National Audit of Cancer Diagnosis in Primary Care. Royal College of General Practitioners, London, UK.

Schiff GD, Puopolo AL, Huben-Kearney A, Yu W, Keohane C, McDonough P, Ellis BR, Bates DW, Biondolillo M (2013) Primary care closed claims experience of Massachusetts malpractice insurers. JAMA Intern Med 173: 2063-2068.

Scott SE, Walter FM, Webster A, Sutton S, Emery J (2013) The model of pathways to treatment: conceptualization and integration with existing theory. Br J Health Psychol 18: 45-65.

Shapley M, Mansell G, Jordan JL, Jordan KP (2010) Positive predictive values of $\geq 5 \%$ in primary care for cancer: systematic review. Br J Gen Pract 60 e366-e377.

Singh H (2014) Helping organizations with defining diagnostic errors as missed opportunities in diagnosis. Jt Comm J Qual Patient Saf 40: 99-101.

Singh H, Daci K, Petersen LA, Collins C, Petersen NJ, Shethia A, El-Serag HB (2009a) Missed opportunities to initiate endoscopic evaluation for colorectal cancer diagnosis. Am J Gastroenterol 104: 2543-2554.

Singh H, Giardina TD, Meyer AN, Forjuoh SN, Reis MD, Thomas EJ (2013a) Types and origins of diagnostic errors in primary care settings. JAMA Intern Med 173: 418-425.

Singh H, Giardina TD, Petersen LA, Smith MW, Paul LW, Dismukes K, Bhagwath G, Thomas EJ (2012a) Exploring situational awareness in diagnostic errors in primary care. BMJ Qual Saf 21: 30-38.
Singh H, Graber ML, Kissam SM, Sorensen AV, Lenfestey NF, Tant EM, Henriksen K, LaBresh KA (2012c) System-related interventions to reduce diagnostic errors: a narrative review. BMJ Qual Saf 21: 160-170.

Singh H, Hirani K, Kadiyala H, Rudomiotov O, Davis T, Khan MM, Wahls TL (2010) Characteristics and predictors of missed opportunities in lung cancer diagnosis: an electronic health record-based study. J Clin Oncol 28: 3307-3315.

Singh H, Khan R, Giardina TD, Paul LW, Daci K, Gould M, El-Serag H (2012b) Postreferral colonoscopy delays in diagnosis of colorectal cancer: a mixed-methods analysis. Qual Manag Health Care 21: $252-261$.

Singh H, Sethi S, Raber M, Petersen LA (2007) Errors in cancer diagnosis: current understanding and future directions. J Clin Oncol 25(31): 5009-5018.

Singh H, Sittig DF (2014) Were my diagnosis and treatment correct? No news is not necessarily good news. J Gen Intern Med 29: 1087-1089.

Singh H, Sittig DF (2015) Advancing the science of measurement of diagnostic errors in healthcare: the Safer Dx framework. BMJ Qual Saf 24(2): 103-110.

Singh H, Spitzmueller C, Petersen NJ, Sawhney MK, Sittig DF (2013b) Information overload and missed test results in electronic health record-based settings. JAMA Intern Med 173: 702-704.

Singh H, Thomas EJ, Mani S, Sittig D, Arora H, Espadas D, Khan MM, Petersen LA (2009b) Timely follow-up of abnormal diagnostic imaging test results in an outpatient setting: are electronic medical records achieving their potential? Arch Intern Med 169: $1578-1586$

Sittig DF, Singh H (2010) A new sociotechnical model for studying health information technology in complex adaptive healthcare systems. Qual Saf Health Care 19(Suppl 3): i68-i74.

Smith MW, Ash JS, Sittig DF, Singh H (2014) Resilient practices in maintaining safety of health information technologies. J Cogn Eng Decis Mak 8(3): 265-282.

Stapley S, Sharp D, Hamilton W (2006) Negative chest X-rays in primary care patients with lung cancer. Br J Gen Pract 56: 570-573.

Vedsted P, Olesen F (2011) Are the serious problems in cancer survival partly rooted in gatekeeper principles? An ecologic study. Br J Gen Pract 61: e508-e512.

Vincent C, Moorthy K, Sarker SK, Chang A, Darzi AW (2004) Systems approaches to surgical quality and safety: from concept to measurement. Ann Surg 239: 475-482.

Walter F, Webster A, Scott S, Emery J (2012) The Andersen Model of Total Patient Delay: a systematic review of its application in cancer diagnosis. $J$ Health Serv Res Policy 17: 110-118.

Zwaan L, de Bruijne M, Wagner C, Thijs A, Smits M, van der Wal G, Timmermans DR (2010) Patient record review of the incidence, consequences, and causes of diagnostic adverse events. Arch Intern Med 170: $1015-1021$.

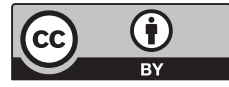

This work is licensed under the Creative Commons Attribution 4.0 International License. To view a copy of this license, visit http://creativecommons.org/licenses/by/4.0/ 